\title{
O Modelo Simples de Leitura Explica a Compreensão Leitora no Português?
}

\section{Does The Simple View of Reading Explain Reading Comprehension in Portuguese?}

\section{El Modelo Simples de la Lectura Explica la Comprensión de la Lectura en Portugués?}

\author{
Marcia Oliveira \\ Universidade Salgado de Oliveira - UNIVERSO, Niterói, Rio de Janeiro, Brasil \\ Márcia Maria Peruzzi Elia da Mota \\ Universidade Salgado de Oliveira - UNIVERSO, Niterói, Rio de Janeiro, Brasil
}

\begin{abstract}
RESUMO
No presente estudo foi investigada a pertinência do Modelo Simples de leitura para explicar a compreensão leitora no português do Brasil em crianças do Ensino fundamental. Participaram desta pesquisa 50 crianças, estudantes do $2^{\circ}$ e $4^{\circ}$ anos do ensino fundamental de uma escola pública, sendo 23 do $2^{\circ}$ ano com idade entre 7 a 9 anos e 27 do 40 ano, com idade entre 9 a 13 anos. Na pesquisa, foram aplicados o Teste de Desempenho Escolar, o Teste de Cloze e de Compreensão de Leitura da PROLEC, leitura de palavras, decisão lexical, leitura de pseudopalavras e de compreensão oral, ambos subtestes da PROLEC. O resultado das correlações e regressões lineares mostraram a importância dos dois componentes do modelo: compreensão oral e reconhecimento de palavras para compreensão de leitura e que estes, contribuem também de forma independentes para a compreensão de leitura.
\end{abstract}

Palavras chave: leitura, compreensão de leitura, reconhecimento de palavras.

\begin{abstract}
The pertinence of the Simple View was investigated in this study in order to explain reading comprehension in Brazilian Portuguese amongst children attending Elementary education. Fifty children look part in the study, students of years 2 and 4 of elementary education from a state school, 23 of which were from year 2, aged between 7 and 9 and 27 from year 4, aged between 9 and 13. During the course of the research conducted, the following tests were applied: the School Performance Test (TDE), the PROLEC, a Cloze test, and Reading Comprehension test, word reading, lexical decision, pseudo-word reading and oral comprehension, subtests from PROLEC. In short, the result of the correlations and linear regressions showed the importance components of the model, oral comprehension and word recognition as well as the fact that these independently contribute to reading comprehension.
\end{abstract}


Keywords: reading, reading comprehension, word recognition.

\begin{abstract}
RESUMEN
En este estudio se investigó la pertinencia del modelo simple para explicar la comprensión lectora en portugués de Brasil entre niños del Ensino fundamental, o Educación primaria. 50 niños participaron del estudio, estudiantes de $2^{\circ}$ y $4^{\circ}$ de primaria de una escuela pública, 23 de los cuales eran de $2^{\circ}$, con edades comprendidas entre 7 y 9 y 27 de 40 , con edades entre 9 y 13. En el estudio se aplicó el test de desempeño escolar, el Test de Cloze y de Comprensión Lectora de PROLEC, lectura de palabras, decisión léxica, lectura de pseudopalabras y comprensión oral, ambos subtestes de PROLEC. En definitiva, el resultado de las correlaciones y regresiones lineares demostró la importancia de los dos componentes del modelo, lectura de palabras y comprensión oral además de que estos contribuyen independientemente hacia la comprensión lectora.
\end{abstract}

Palabras clave: lectura, comprensión lectora, reconocimiento de palabras.

A compreensão de leitura é o principal objetivo de se aprender a ler na escola. Sem ela as crianças têm dificuldades em diversas atividades, não só as escolares, mas até nas vinculadas às tarefas do dia a dia que em geral envolvem a leitura, especialmente nas sociedades industrializadas (Solé, 1992; Cagliari, 1994). Compreender quais as estratégias cognitivas que estão envolvidas no processo desta aquisição pode ajudar a criar intervenções que sejam mais efetivas na promoção do aprendizado da leitura. Adicionalmente, pode ajudar na criação de instrumentos de avaliação e no diagnóstico precoce dos problemas de leitura.

A literatura na área tem descrito diversos modelos que tentam explicar o processo de compreensão de leitura (Kintsch, 1998; Perfetti, 1985). O Modelo de Kintch propõe que a compreensão do texto ocorre em diferentes níveis de análise, que se integram para promover a compreensão. O Modelo da eficiência verbal de Perfetti, por sua vez, sugere que a decodificação eficiente é necessária para que sobre espaço de processamento cognitivo, necessário para as operações cognitivas que ocorrem em outros níveis análise (sintáticosemântico, inferenciais) na compreensão do texto.

Um modelo que tem sido revisitado, recentemente, é o Modelo Simples de Leitura (Florit \& Cain, 2011; Adlof, Catts \& Little, 2006; Kirby \& Savage, 2008; Correa \& Mousinho, 2013). Proposto inicialmente por Gough e Tunmer (1986) e Hoover e Gough (1990), esse modelo oferece um arcabouço simples para explicar o processo de compreensão de texto, como o próprio nome diz. No presente trabalho, investiga-se a pertinência do Modelo Simples para explicar a compreensão leitora no português do Brasil em crianças do Ensino Fundamental.

O Modelo Simples propõe que a compreensão de leitura resulta do produto de duas habilidades: decodificação e compreensão 
linguística. Gough e Tunmer (1986) destacaram que, neste modelo a compreensão de leitura $(C L)$ é vista como o produto da compreensão oral $(C O) \times$ decodificação $(D)$, logo $C L=C O \times D$. Decodificação refere-se à habilidade de ler palavras. Isto pode ocorrer pela leitura de pseudopalavras impressas por aplicação das regras de correspondência grafema-fonema ou pelo reconhecimento de palavras pela rota lexical. A compreensão oral (ou linguística) referese à capacidade de interpretar frases e discursos apresentados oralmente. De acordo com o modelo, o desenvolvimento da leitura não ocorreria na ausência de qualquer uma das duas habilidades mencionadas no modelo. Dificuldades encontradas pela criança na decodificação trariam, como consequência imediata, dificuldades na compreensão leitora, da mesma forma que dificuldades na compreensão linguística também dificultariam a compreensão do texto.

Adlof, Catts e Little (2006) revisaram estudos sobre compreensão de texto e mostram que os componentes do Modelo Simples de Leitura explicam grande parte da variação na compreensão de textos (entre $45 \%$ e $85 \%$ ) dos indivíduos. Assim, é um bom ponto de partida para entendermos os componentes cognitivos da compreensão de texto, uma vez que esses dois componentes são responsáveis em média por mais da metade da variância em leitura.

A literatura aponta, também, para o fato de que o Modelo Simples de Leitura constitui uma ferramenta útil para professores/educadores, porque permite que se inclua tanto os aspectos ligados as práticas de ensino da leitura, ligados a alfabetização, quanto os aspectos ligados ao letramento. Ou seja, este modelo relaciona tanto os aspectos da decodificação quanto os aspectos da compreensão linguística. 0 Modelo é útil para conceituar essas grandes habilidades no planejamento de ensino e aprendizagem. E tem sido utilizado como uma estrutura para pensar sobre a distinção entre as dificuldades que afetam a decodificação e as que afetam a compreensão oral (Kirby \& Savage, 2008).

Uma observação que precisa ser feita é que o Modelo Simples, não apresenta uma contribuição equivalente dos seus componentes para leitura ao longo do desenvolvimento. O quanto cada uma dessas habilidades $(\mathrm{D} \times \mathrm{CO})$ contribui para compreensão leitora dependerá de vários fatores: a idade de aquisição das crianças, o tipo de ortografia a ser investigada e o tipo de teste de compreensão leitora utilizado.

Em relação à idade de aquisição as pesquisas mostram que a contribuição relativa destes componentes do Modelo Simples de Leitura é sujeita às mudanças ao longo do tempo. Nas séries iniciais, a compreensão da leitura é principalmente explicada pela capacidade de leitura de palavras. Na medida em que os alunos alcançam leituras mais complexas nos anos posteriores, a contribuição da compreensão 
linguística aumenta na compreensão, enquanto que a contribuição de leitura de palavras diminui, isto também, porque nesta etapa os processos de reconhecimento de palavras estão mais automatizados (Georgiou, Das \& Hayward, 2009; Johnston \& Kirby, 2006; Savage, 2006; Adlof et al., 2006),

Em relação ao tipo de ortografia os estudos mostram que para línguas mais transparentes como o Finlandês, que apresenta regras de correspondência entre fonemas-grafemas totalmente regulares, a decodificação é adquirida mais rapidamente, portanto a compreensão linguística pode ter um peso mais importante desde mais cedo no desenvolvimento da leitura. O português é colocado no meio do espectro quando tratamos da regularidade ortográfica (Seymour, Aro \& Erskine, 2003). É uma ortografia pouco transparente em relação ao espanhol e italiano nas quais a correspondência fonema/grafema é quase exata, mas com uma estrutura fonológica mais transparente do que $o$ inglês e do que o francês, que são consideradas quase opacas. Neste caso, questiona-se: a compreensão linguística teria um peso menor ou maior do que o reconhecimento de palavras na compreensão leitora?

Florit e Cain (2011) realizaram uma meta-análise para comparar a influência da decodificação e compreensão linguística (compreensão oral) na compreensão de leitura entre os leitores iniciantes de Inglês que, como já estabelecemos, é uma língua opaca em relação a outras ortografias mais transparentes (grego, holandês, francês e finlandês). Eles investigaram vinte estudos com leitores iniciantes em Inglês, e treze em crianças que falam outras línguas mais transparentes. As autoras identificaram que durante os primeiros anos em ortografias transparentes, a correlação entre a precisão de leitura e compreensão de leitura foi muito menor do que para os alunos de língua Inglesa.

O português está classificado segundo Seymour et al. (2003) como uma ortografia moderadamente opaca e de estrutura silábica simples. Assim, a complexidade de algumas correspondências entre letra e som caracterizam a língua portuguesa como uma ortografia intermédia entre as línguas alfabéticas. Quanto mais opaco for 0 sistema linguístico mais irregularidades irão existir. Neste sentido, estudar a língua portuguesa contribuirá para investigar se o Modelo Simples se ajusta a línguas alfabéticas como o português, oferecendo evidências adicionais para sustentar esse modelo de compreensão de texto. Se esse for o caso as contribuições dos componentes da leitura devem ser coerentes com a complexidade ortográfica da língua.

Em resumo, pretende-se investigar se os componentes do Modelo Simples no português podem ajudar a corroborar as hipóteses já levantadas a respeito deste Modelo como modelo explicativo da aquisição de leitura em línguas alfabéticas. Como implicações o estudo pode ajudar no diagnóstico e na elaboração de programas de remediação das dificuldades de compreensão de leitura. Assim, os 
objetivos do presente estudo são: 1) verificar se o modelo simples explica a compreensão de leitura no português. Se este é o caso, espera-se que o reconhecimento de palavras e compreensão linguística contribuam para a compreensão leitora de crianças brasileiras. 2) verificar se as características ortográficas afetam a contribuição dos diferentes componentes do Modelo. Como o português está no meio do espectro ortográfico, não é clara ainda a importância dos diferentes componentes para compreensão leitora. Pode-se esperar contribuições equivalentes dos dois componentes para leitura no português.

\section{Método}

\section{Participantes}

Cinquenta crianças do $2^{\circ}$ e $4^{a}$ ano do ensino fundamental, sendo 23 do $2^{\circ}$ ano com idades entre 84 meses a 108 meses; $M=101,47$ meses, $D P=16,64$ meses e 27 do $4^{\circ}$ ano, com idades entre 108 meses a 156 meses; $M=126,14$ meses, $D P=11,53$ meses de uma escola pública no Município de São Gonçalo, região metropolitana do Rio de Janeiro. Quatro crianças não completaram o teste de Cloze porque não estavam presentes no dia. O critério de inclusão foi a assinatura, pelos seus responsáveis, do Termo de consentimento livre e esclarecido (TCLE). A pesquisa foi aprovada pelo Comitê de Ética e Pesquisa com o número de registro 085.3.2012.

\section{Instrumentos}

Avaliação da Leitura: Subteste de Leitura e da Escrita do Teste de Desempenho escolar - TDE (Stein, 1994). TDE visa a verificar o desempenho global da criança e seu desempenho isolado em leitura e escrita. Para este estudo, foram aplicados apenas os subtestes de leitura em que as crianças devem ler, em voz alta, um conjunto de 70 palavras isoladas do contexto e distribuídas em uma folha. A tarefa apresenta evidência de validade de critério e bom índice de consistência interna $(a>0,70)$. O subteste foi administrado e corrigido de acordo com as instruções e procedimentos especificados no manual. Assim para cada acerto, foi dado um ponto e para cada erro, zero ponto, perfazendo um total de 70 pontos. Para fins de nossa análise foram usados o número de erros.

Teste de Cloze (Oliveira, Burochovich \& Santos, 2007): O teste consiste na organização de dois pequenos textos " $A$ princesa $e$ fantasma" e "Uma vingança infeliz" no qual se suprimem alguns vocábulos e se pede ao aluno que preenchpra os espaços com palavras que melhor completem o sentido do texto. O Máximo de 
acertos possíveis para a tarefa usado foi de 30 pontos. Uma vez que, para cada acerto foi dado um ponto.

Avaliação dos Processos de Leitura e Compreensão Oral - PROLEC adaptação brasileira por Capellini, Oliveira e Cuetos (2010), desenvolvida na Espanha por Cuetos, Rodrigues e Ruano (1996). Foi utilizada uma medida que compõe a Avaliação dos Processos de Leitura da PROLEC para avaliação da medida de compreensão oral.

A PROLEC apresenta provas de leitura de palavras com a finalidade de verificar o funcionamento das duas rotas (a fonológica e a visual ou léxica) de reconhecimento de palavras e seus subprocessos e também a compreensão oral das crianças. Na prova de decisão lexical, a criança deve reconhecer apenas palavras reais em uma lista de palavras reais e inventadas, independentemente de ser capaz de lê-las. Nas provas de leitura de palavras, leitura de pseudopalavras, o objetivo é verificar o desenvolvimento do reconhecimento de palavras. A criança deve realizar a leitura de palavras reais e inventadas, sendo que na primeira prova foi medida a capacidade do escolar ler palavras reais e, na segunda, a capacidade para ler palavras inventadas, de diferentes complexidades silábicas, organizadas com as sequências ortográficas CCV, VC, CVC, CVV, CCVC e CVVC. Na quarta prova, o objetivo é analisar o grau de desenvolvimento da compreensão oral das crianças. Computou-se o número de acertos para cada prova. Todas as provas foram utilizadas. Utilizou-se ainda a prova de compreensão de texto dessa bateria.

\section{Procedimento}

Os participantes foram avaliados, individualmente, em uma sala silenciosa dentro da escola cedida pela direção da escola. Foram realizadas cinco sessões, sendo a primeira o teste de desempenho em leitura (TDE leitura), a segunda, 0 teste Cloze, a terceira compreensão de texto e a decisão lexical, a quarta a leitura de palavras e pseudopalavras e a quinta o teste de compreensão oral. As sessões ocorreram sempre em horários de aula, previamente estabelecidos pela coordenação e professores. Os testes duraram aproximadamente 35 minutos.

As crianças foram avaliadas quanto ao TDE e a PROLEC seguindo as instruções do manual. Para o teste de Cloze pediu-se que os participantes lessem a estória até o final, antes de preencher as lacunas. Após, essa leitura, as crianças foram instruídas a lerem novamente o texto e que preenchessem a lacuna com uma palavra somente. 


\section{Resultados}

A Tabela 1 mostra a média e o desvio padrão para o número de acertos na tarefa de Cloze, compreensão de texto, no TDE leitura, nas tarefas de decisão lexical, tarefas de leitura de palavras e pseudopalavras e compreensão oral.

Tabela 1

Média e Desvio Padrão das tarefas do teste Cloze, da tarefa de compreensão de texto, do TDE leitura, da decisão lexical, das tarefas de leitura de palavras e pseudopalavras, e compreensão oral:

\begin{tabular}{|c|c|c|c|c|}
\hline \multicolumn{2}{|c|}{ Serie/ Variáveis } & \multirow{2}{*}{$\begin{array}{l}N \\
23\end{array}$} & \multirow{2}{*}{$\begin{array}{l}\text { Média } \\
1,45\end{array}$} & \multirow{2}{*}{$\begin{array}{l}\text { Desvio Padrão } \\
1,07\end{array}$} \\
\hline $2^{\circ}$ ano & Teste Cloze & & & \\
\hline & Compreensão de texto & 23 & 4,26 & 2,63 \\
\hline & TDE Leitura & 23 & 5,44 & 2,26 \\
\hline & Decisão lexical & 23 & 13,69 & 5,95 \\
\hline & Leitura de palavras & 23 & 12,17 & 6,32 \\
\hline & Leitura de pseudopalavras & 23 & 11,43 & 5,49 \\
\hline & Compreensão oral & 23 & 2,10 & 0,52 \\
\hline \multirow[t]{7}{*}{40 ano } & Teste Cloze & 23 & 2,92 & 0,85 \\
\hline & Compreensão de texto & 27 & 9,66 & 2,55 \\
\hline & TDE Leitura & 27 & 2,53 & 1,59 \\
\hline & Decisão lexical & 27 & 16,40 & 4,74 \\
\hline & Leitura de palavras & 27 & 19,29 & 4,72 \\
\hline & Leitura de pseudopalavras & 27 & 15,55 & 4,79 \\
\hline & Compreensão oral & 27 & 1,72 & 0,44 \\
\hline
\end{tabular}

Nota: Para o TDE se calculou o número de erros.

A Tabela 2 apresenta a Skewness e Kurtosis para as medidas investigadas. Essas medidas permitem avaliar se as distribuições são apropriadas para análises paramétricas. Podemos observar que as variáveis: teste de Cloze, TDE leitura e a compreensão oral apresentam distribuições que fogem o padrão de distribuição normal e, portanto, não podemos utilizar dados paramétricos com essas variáveis. Para procedermos com a utilização da análise dos dados 
com distribuição normal, os dados sofreram transformações para tentar normalizá-la. A transformação escolhida foi a de raiz quadrada.

Tabela 2

Estatísticas descritivas para as distribuições das medidas do Estudo

\begin{tabular}{lcccc}
\hline & \multicolumn{3}{c}{ Assimetria } & \multicolumn{2}{c}{ Curtose } \\
\cline { 2 - 5 } & Estatística & Erro padrão & Estatística & Erro padrão \\
\hline Teste Cloze & 0,81 & 0,35 & $-0,31$ & 0,68 \\
Compreensão de Texto & $-0,21$ & 0,33 & $-1,20$ & 0,66 \\
TDE Leitura & 1,00 & 0,33 & $-0,31$ & 0,66 \\
Decisão Lexical & 0,61 & 0,33 & 0,43 & 0,66 \\
Leitura de Palavras & 0,20 & 0,33 & $-0,70$ & 0,66 \\
Leitura de Pseudopalavras & 0,01 & 0,33 & $-1,31$ & 0,66 \\
Compreensão Oral & $-1,07$ & 0,33 & 1,81 & 0,66 \\
\hline
\end{tabular}

Após a transformação dos dados foi possível normalizar todas as variáveis. Correlações de Pearson exploraram a relação entre as variáveis de reconhecimento de palavras e compreensão oral com as tarefas de compreensão de texto. Os resultados encontram-se na Tabela 3.

Tabela 3

Mostra os Coeficientes das Correlações Bivariadas de Pearson entre os testes de Cloze, compreensão de texto, TDE leitura, leitura de palavras, decisão lexical, leitura de palavras e pseudopalavras e compreensão oral.

\begin{tabular}{|c|c|c|c|c|c|c|}
\hline Variáveis & 2 & 3 & 4 & 5 & 6 & 7 \\
\hline 1 - Teste Cloze & $0,69^{* *}$ & $-0,73^{\mathrm{kn}}$ & $0,40^{\text {*n }}$ & $0,56^{* *}$ & $0,49^{* *}$ & $-0,54^{* 4}$ \\
\hline 2 - Compreensão de texto & & $-0,73^{* 4}$ & $0,64^{* *}$ & $0,79^{-*}$ & $0,70^{* *}$ & $-0,74^{-\cdots}$ \\
\hline 3 - TDE leitura & & & $-0,50^{* *}$ & $-0,65^{* u}$ & $-0,50^{* *}$ & $0,51^{-*}$ \\
\hline 4 - Decisão lexical & & & & $0,90^{* *}$ & $0,89^{* *}$ & $-0,58^{* *}$ \\
\hline 5 - Leitura de palavras & & & & & $0,89^{* *}$ & $-0,60^{* *}$ \\
\hline 6 - Leitura pseudopalavras & & & & & & $-0,56^{* *}$ \\
\hline 7 - Compreensão oral & & & & & & \\
\hline
\end{tabular}

Nota: (**) correlações significativas em nível 0,01 .

As variáveis que sofreram transformações devem ser interpretadas com o sinal invertido 
Como se esperava todas as correlações foram significativas. Em relação à hipótese levantada destacamos as correlações significativas entre o TDE leitura e o CLOZE, $(r=-0,73 * *, p<0,01)$ e o TDE leitura e a tarefa de compreensão de texto $\left(r=-0,73^{* *}, p<0,01\right)$. O TDE sofreu transformações quadráticas, então, o sinal negativo deve ser invertido para interpretarmos essas correlações. Esses resultados indicam que quanto melhor as crianças eram na leitura de palavras, melhor na compreensão de textos.

Esses resultados se repetiram com as outras medidas de leitura de palavras (decisão lexical, leitura de palavras e pseudopalavras). As correlações entre essas medidas e os dois testes de compreensão de texto foram significativas e positivas e variaram entre moderadas e fortes, corroborando a ideia de que o reconhecimento de palavras contribui para compreensão de texto como proposto pelo Modelo Simples.

Em relação à Compreensão Oral (CO), o sinal das correlações também precisa ser invertido para ser interpretado, pois essa variável também sofreu transformações quadráticas. Podemos observar pela Tabela 3 que todas as correlações entre a Compreensão Oral e a Compreensão de Texto foram significativas. Para o Cloze, elas foram moderadas $(r=-0,54 * *, p<0,01)$ e para compreensão de texto forte $(r=-0,74 * *, p<0,01)$. Esses dados sugerem que esse componente do Modelo Simples também contribui para a compreensão de leitura no português do Brasil.

Tendo como objetivo investigar se o reconhecimento de palavras e a compreensão oral contribuem para a compreensão de leitura, análises de regressão foram realizadas. As variáveis dependentes inseridas na análise de regressão para a compreensão da leitura foram: teste Cloze e a compreensão de texto da PROLEC.

Análises de regressões foram realizadas com o objetivo de verificar se, como prediz o Modelo Simples, as variáveis reconhecimento de palavras (teste de desempenho em leitura, decisão lexical, leitura de palavras e leitura de pseudopalavras) e compreensão oral contribuem para a leitura independente da idade. Os resultados das regressões encontram-se na Tabela 4. 
Tabela 4

Análise de regressão, sendo as variáveis-critério o teste Cloze e a Compreensão de Texto e como variáveis explicativas a idade, as medidas de reconhecimento de palavras e a compreensão oral.

\begin{tabular}{|c|c|c|c|c|c|c|c|c|c|c|}
\hline \multirow[t]{3}{*}{ Variáveis } & \multicolumn{4}{|c|}{ Variável Critério } & \multirow{3}{*}{$\begin{array}{l}\mathrm{R} \\
\text { Square } \\
\text { Change }\end{array}$} & \multicolumn{4}{|c|}{ Variável Critério } & \multirow{3}{*}{$\begin{array}{l}\mathrm{R} \\
\text { Square } \\
\text { Change }\end{array}$} \\
\hline & \multicolumn{4}{|c|}{ Teste Cloze } & & \multicolumn{4}{|c|}{ Compreensão de texto } & \\
\hline & Beta & R2 & $\mathrm{F}$ & Sig. $<$ & & Beta & $\mathrm{R} 2$ & $\mathrm{~F}$ & Sig. $<$ & \\
\hline Idade (meses) & 0,55 & 0,28 & 19,28 & $0,01^{*}$ & 0,30 & 0,37 & 0,12 & 7,66 & 0,08 & 0,13 \\
\hline TDE Leitura & $-0,62$ & 0,63 & 40,06 & $0,01^{*}$ & 0,34 & $-0,68$ & 0,53 & 29,26 & $0,01^{*}$ & 0,41 \\
\hline Compreensão & $.0,26$ & 0,68 & 33,05 & $0,01 *$ & 0,05 & $-0,51$ & 0,73 & 46,04 & $0,01 *$ & 0,19 \\
\hline \multicolumn{11}{|l|}{ oral } \\
\hline Compreensão & 0,49 & 0,52 & 25,56 & $0,01^{*}$ & 0,23 & $-0,71$ & 0,62 & 42,04 & $0,01^{*}$ & 0,15 \\
\hline \multicolumn{11}{|l|}{ oral } \\
\hline TDE leitura & $-0,48$ & 0,68 & 33,05 & $0,01^{*}$ & 0,15 & $-0,40$ & 0,73 & 46,04 & $0,01^{*}$ & 0,10 \\
\hline Decisão lexical & 0,39 & 0,43 & 18,36 & $0,01^{*}$ & 0,15 & 0,63 & 0,52 & 27,59 & $0,01^{*}$ & 0,40 \\
\hline Compreensão & $-0,39$ & 0,52 & 17,81 & $0,01 *$ & 0,09 & $-0,51$ & 0,69 & 38,36 & $0,01^{*}$ & 0,17 \\
\hline \multicolumn{11}{|l|}{ oral } \\
\hline Compreensão & $-0,49$ & 0,52 & 25,56 & $0,01^{*}$ & 0,23 & $-0,71$ & 0,62 & 42,04 & $0,01^{*}$ & 0,50 \\
\hline \multicolumn{11}{|l|}{ oral } \\
\hline Decisão lexical & 0,16 & 0,52 & 17,81 & 0,213 & 0,17 & 0,33 & 0,69 & 38,36 & $0,001 * *$ & 0,07 \\
\hline Leitura de & 0,46 & 0,48 & 21,87 & $0,01^{*}$ & 0,20 & 0,75 & 0,64 & 46,27 & $0,01^{*}$ & 0,52 \\
\hline \multicolumn{11}{|l|}{ Palavras } \\
\hline Compreensão & $-0,34$ & 0,54 & 19,02 & $0,01 *$ & 0,07 & $-0,43$ & 0,76 & 54,23 & $0,01^{*}$ & 0,11 \\
\hline oral & & & & & & & & & & \\
\hline $\begin{array}{l}\text { Compreensão } \\
\text { oral }\end{array}$ & $.0,49$ & 0,52 & 25,56 & $0,01^{*}$ & 0,23 & $-0,71$ & 0,62 & 42,04 & $0,01^{*}$ & 0,50 \\
\hline Leitura de & 0,16 & 0,52 & 17,81 & 0,213 & 0,17 & 0,33 & 0,69 & 38,36 & $0,001 * *$ & 0,07 \\
\hline Palavras & & & & & & & & & & \\
\hline $\begin{array}{l}\text { Leitura de } \\
\text { pseudopalavras }\end{array}$ & 0,43 & 0,47 & 21,10 & $0,01^{*}$ & 0,08 & $-0,49$ & 0,72 & 43,40 & $0,01^{*}$ & 0,43 \\
\hline $\begin{array}{l}\text { Compreensão } \\
\text { oral }\end{array}$ & $-0,35$ & 0,54 & 19,19 & $0,01^{*}$ & 0,08 & $-0,49$ & 0,72 & 43,40 & $0,01^{*}$ & 0,16 \\
\hline $\begin{array}{l}\text { Compreensão } \\
\text { oral }\end{array}$ & $-0,49$ & 0,52 & 25,56 & $0,01^{*}$ & 0,23 & $-0,71$ & 0,62 & 42,04 & $0,01^{*}$ & 0,50 \\
\hline $\begin{array}{l}\text { Leitura da } \\
\text { pseudopalavras }\end{array}$ & 0,35 & 0,59 & 22,90 & $0,01^{*}$ & 0,07 & 0,44 & 0,74 & 47,62 & $0,01^{*}$ & 0,11 \\
\hline
\end{tabular}

Nota: ${ }^{*} p<0,01 /{ }^{* * *} p<0,001$ 
Quando o teste Cloze é a variável de critério e a primeira variável a entrar no modelo é a idade, e a segunda, uma das medidas de reconhecimento de palavras, os escores do TDE, a compreensão oral ainda contribui com cerca de $5 \%$ da variância da compreensão de texto. A variável desempenho em leitura (TDE), explica $34 \%$ da variância para esta habilidade. Quando se inverte a ordem, o Cloze continua como variável de critério e a compreensão oral entra como segundo passo na análise, a idade, como primeiro, os resultados indicam que a contribuição de desempenho em leitura (TDE) é de $15 \%$ e a compreensão oral explica $23 \%$ do modelo. Esses resultados foram significativos.

As regressões que tiveram o teste de compreensão de leitura como variável de critério, a idade como primeiro passo, as variáveis desempenho em leitura (TDE), como segundo passo, e a compreensão oral como passo seguinte, verifica-se que o desempenho na leitura de palavras explica $41 \%$ do modelo e a compreensão oral contribui com 19\%, Ao inverter a entrada variável compreensão oral com as variáveis desempenho na leitura (TDE) constatou-se que a compreensão oral contribui com $50 \%$ e o TDE apenas $10 \%$. Esses resultados foram significativos.

Quando uma das variáveis explicativas do modelo é a decisão lexical, tendo o Teste de Cloze como variável critério, e a idade continua sendo a variável de controle, entrando no modelo como primeiro passo, e inserimos no modelo a decisão lexical seguida pela compreensão oral, os resultados apontam que a decisão lexical contribui com $15 \%$ e a compreensão oral com $9 \%$ da variância. Invertendo as inscrições das variáveis explicativas, sendo a idade a primeira variável ser inserida como controle, a compreensão oral a segunda e a terceira variável a decisão lexical, a contribuição da compreensão oral foi de $23 \%$ e a decisão lexical explicou $17 \%$ do modelo. Esses resultados não foram significativos, para contribuição da decisão lexical para o Cloze. Mas, quando a variável critério é a compreensão de texto, em primeiro momento sendo a decisão lexical inserida antes da compreensão oral, observa-se na Tabela 4, que os resultados são significativos, a decisão lexical aparece com contribuição de $40 \%$ e a compreensão oral $17 \%$ da variância em leitura. E quando invertidas, sendo-a compreensão oral e depois a decisão lexical, a compreensão oral explica $50 \%$ da variância e a decisão lexical contribui somente $7 \%$ da variância, mas a contribuição foi significativa.

Considerando a variável critério o Teste Cloze, inserindo no modelo a idade como controle e inserindo como segundo a variável leitura de palavras e a terceira variável compreensão oral, o modelo tem a contribuição de $20 \%$ da leitura de palavra e $11 \%$ de compreensão oral para a compreensão de texto. Invertendo os passos entre as variáveis explicativas, sendo a compreensão oral colocada como 
segundo passo e a leitura de palavras como terceiro, observa-se que a compreensão oral passa a explicar $23 \%$ da variância de compreensão de texto enquanto que a leitura de palavras contribui com $17 \%$ da variância no modelo.

Quando a variável critério passa a ser a compreensão de texto e a primeira variável de controle a idade, seguida da variável explicativa leitura de palavras e, como terceiro passo a compreensão oral, o modelo passa a ter $52 \%$ de contribuição da leitura de palavras e continua a ter $11 \%$ da contribuição da compreensão oral. Quando a compreensão oral é inserida no modelo primeiro do que a leitura de palavras, o modelo passa a ser explicado por $50 \%$ da compreensão oral e apenas $7 \%$ da leitura de palavras. Esses resultados também foram significativos.

No modelo de regressão, considerando como variável critério o Teste Cloze, tendo a idade como controle e, o segundo passo inserido a variável leitura de pseudopalavras, seguida da compreensão oral, a contribuição da leitura de pseudopalavras foi de $19 \%$ e a compreensão oral contribuiu com $8 \%$. Quando estas variáveis leitura e compreensão são invertidas no modelo, a compreensão oral passa a contribuir com $23 \%$ e a leitura com $7 \%$ da variância. Se a variável critério para essas duas variáveis passa a ser a compreensão de texto, seguida da idade como controle e a leitura de pseudopalavras inserida como próximo passo, seguida de seguida a compreensão oral, observa-se que a leitura de pseudopalavras passa a explicar $43 \%$ do modelo e a compreensão oral explica $16 \%$ da variância. Quando invertidos esses passos, sendo a compreensão oral inserida antes da leitura de pseudopalavras, o modelo a compreensão oral passa a explicar $50 \%$ da variação na compreensão de texto e a leitura de pseudopalavras $11 \%$. Todas as contribuições foram estatisticamente significativas.

\section{Discussão}

A primeira pergunta do presente estudo foi se o Modelo Simples de Leitura explica a compreensão de leitura no português do Brasil. Os resultados apontam que sim. As medidas de decodificação e compreensão linguística correlacionaram de forma positiva e significativa com a compreensão de leitura medida pelo teste da PROLEC e de CLOZE no que diz respeito a todas as medidas de reconhecimento de palavras: leitura (TDE), decisão lexical, leitura de palavras, leitura de pseudopalavras e com a compreensão oral. Quanto melhor a compreensão oral e o reconhecimento de palavras melhor era a compreensão de textos dos estudantes.

Além disso, quando olhamos a contribuição da idade e os dois componentes investigados, reconhecimento de palavras e 
compreensão oral nos modelos de regressão, vemos que essas variáveis explicaram, entre $50 \%$ e $70 \%$ da variação da compreensão de texto nos dois testes usados. Esses resultados mostram que grande parte da variação da compreensão de leitura no português é composta por essas variáveis e são compatíveis com o que já foi revisado na literatura em outras línguas. Cabe ressaltar que, quando a idade das crianças é controlada, os dois componentes continuam a explicar a compreensão de leitura de forma significativa.

A segunda questão do estudo testou a hipótese de que esses dois componentes predizem significativamente a compreensão leitora no português e verificou a contribuição de cada um deles para leitura. Estes resultados foram testados através de regressões lineares, controlando a idade dos participantes como primeiro passo da análise. Aqui obtivemos pequenas diferenças quando o teste foi 0 Cloze ou quando foi o teste de compreensão de leitura da PROLEC.

Para todos os testes, não importando a ordem de entrada dos componentes da leitura, após controlar a idade dos participantes, os resultados mostraram contribuições significativas. As únicas exceções foram para o teste de Cloze que mostrou que quando se controla a idade e a compreensão oral, a contribuição da leitura de palavras e a decisão lexical deixam de ser significativas. Os resultados das regressões, quando a variável que avaliava reconhecimento de palavras exigem um maior processamento fonológico (leitura de pseudopalavras), se mantêm significativos. Assim, uma hipótese que pode ser levantada é que é possível, que os resultados sejam devidos ao tipo de estratégia que as crianças usam a ler palavras pela via lexical. Elas podem na leitura por essa via usar informações sintáticosemânticas que são comuns a compreensão oral ao ler essas palavras e, portanto, quando a variância comum a essas estratégias é controlada, sua influência diminui.

Precisamos olhar também, como os diferentes componentes contribuem para compreensão de leitura, não apenas a pertinência do Modelo para explicar a compreensão de leitura no português do Brasil. Em seu modelo Gough e Tunmer (1986) propuseram uma contribuição equivalente dos dois componentes, ou seja, tanto o reconhecimento de palavras quanto a compreensão oral deveriam contribuir com cerca de $50 \%$ da variância da compreensão leitora. Os resultados mostraram que para as análises feitas com a PROLEC os resultados se aproximavam a $50 \%$ da variância dependendo da variável que entrava primeiro na análise, indicando que os dois componentes, compreensão oral e reconhecimento de palavras, contribuem para a compreensão leitora no português do Brasil, como previsto pelo Modelo Simples. A única exceção foi para a leitura de pseudopalavras. Vamos lembrar que pseudopalavras só podem ser lidas por correspondência letra e som e portanto, exigem uma estratégia mais forte de reconhecimento de palavras do que de 
aspectos de integração da linguagem oral. Esse fato pode explicar a diferença nesse resultado.

Para o Cloze as contribuições dos componentes foram relativamente equivalentes também. No entanto, menores que na PROLEC. Kirby e Savage (2008) criticaram o Cloze por não equilibrar adequadamente os componentes do Modelo Simples, sendo muito influenciado pela leitura de palavras. Olhando as variâncias na Tabela 4, vemos que há uma pequena vantagem do reconhecimento de palavras para esse teste. Nos casos; em que se avalia a contribuição do reconhecimento de palavras que são lidas por estratégias lexicais o resultado deixa de ser significativo para contribuição dessas variáveis. É preciso explorar melhor quais as estratégias usadas pelas crianças para realizar 0 teste de Cloze e sua adequação para avaliar a compreensão de leitura. Estudos posteriores aos de Gough e Tunmer (1986), revisados na introdução deste trabalho como os de Florit e Cain (2011) podem lançar luz em alguns dos nossos resultados. Florit e Cain também testaram a validade do Modelo Simples em leitores nos anos iniciais para o inglês e outras ortografias transparentes. Em seus estudos, mostraram que os componentes de leitura do Modelo Simples possuem diferentes influências na compreensão leitora durante o desenvolvimento da leitura nos anos iniciais em diferentes ortografias. De acordo com este estudo, em ortografia menos transparente, como o inglês, a decodificação é mais influente, mas em ortografias mais transparentes, a compreensão oral possui uma contribuição mais importante no desenvolvimento da leitura.

Os dois componentes pareceram contribuir, em geral, de forma equivalente no presente estudo. Os nossos resultados apontam contribuição independente de cada elemento do Modelo Simples de leitura. O reconhecimento de palavras contribuiu para a compreensão leitora independente da compreensão oral. Da mesma forma, a compreensão oral contribuiu para a compreensão de leitura independente do reconhecimento de palavras. Nesse sentido, nossos resultados apontam para o português como uma ortografia que se localiza no meio do espectro ortográfico, como foi proposto por Seymour et al. (2003). Corroboram a proposta do espectro ortográfico de Seymour e ampliam os achados de Florit e Cain (2011) incorporando o português entre as línguas que investigaram o Modelo Simples.

O estudo apresentou limitações como o tamanho da amostra. A junção dos dois grupos se justifica uma vez que crianças de $2^{\circ}$ e $4^{\circ}$ ano, já se encontram alfabetizadas e nessa etapa entrando no estágio ortográfico (Mota, 1996). Um último ponto que precisa ser levantado, é que nessa faixa etária, o sistema alfabético já está consolidado e portanto, a influência da leitura de palavras pode ter sido menor. 


\section{Conclusão}

A compreensão de leitura é um construto que envolve processos complexos, mas o Modelo Simples aponta para dois componentes principais, o reconhecimento de palavras e a compreensão oral como contribuindo para a maior parcela da variância na compreensão de textos escritos. Os resultados indicaram que o Modelo Simples de fornece um quadro explicativo para a compreensão de leitura em diversos idiomas e, também para o português do Brasil. O Modelo Simples pode constituir uma ferramenta útil para professores e pesquisadores. Nossos resultados sugerem que os educadores devem desenvolver atividades que melhorem o domínio dos componentes do Modelo Simples, para melhorar a compreensão de leitura, também, devem investigar o papel dos componentes do Modelo Simples nas dificuldades encontradas pelos estudantes. Mais estudos são necessários, com amostras maiores e em diferentes momentos do processo de escolarização para termos uma compreensão melhor de como esses componentes funcionam no português do Brasil.

\section{Referências}

Adlof, S. M., Catts, H. W., \& Little, T. D. (2006). Should the simple view of reading include a fluency component? Reading and Writing, 19, 933-958. doi: 10.1007/s11145-006-9024.

Capellini S. A., Oliveira A. M., \& Cuetos F. (2010). PROLEC: provas de avaliação dos processos de leitura. São Paulo: Casa do Psicólogo.

Cagliari, L. C. (1994). Alfabetização e linguística (2 ed.). São Paulo: Scipione.

Correa, J. \& Mousinho, R. (2013). Por um modelo simples de leitura, porém não tão simples assim. In M. Mota \& A. Spinillo (Orgs). Compreensão de textos (pp. 77-100). São Paulo: Casa do Psicólogo.

Cuetos, F., Rodrigues, B., \& Ruano, E. (1996). Evaluación de lós procesos lectores. Madrid: TEA Ediciones.

Florit, E. \& Cain, K. (2011). The simple view of reading: is it valid for different types of alphabetic orthographies? Educational Psychology Review, 23, 553-576. doi: 10.1007/s10648-0119175-6.

Georgiou, G. K., Das, J. P. \& Hayward, D. (2009). Revisiting the "Simple View of Reading" in a Group of Children With Poor Reading Comprehension. Journal of Learning Disabilities, 42, 76-86. 
Gough, P. B., \& Tunmer, W. E. (1986). Decoding, reading \& reading disability. Remedial and Special Education, 7, 6-10. doi: $10.1177 / 074193258600700104$

Hoover, W. A., \& Gough, P. B. (1990). The simple view of reading. Reading and Writing: An Interdisciplinary Journal, 2, 127-160.

Hoover, W. A., \& Tunmer, W. E. (1993). The components of reading. In G. Thompson, W. Tunmer, \& T. Nicholson (Orgs.), Reading acquisition processes (pp.1-19). Clevedon, England: Multilingual Matters.

Johnston, T. C. \& Kirby, J. R. (2006). The contribution of naming speed to the simple view of reading. Reading and Writing, 19, 339-361.

Kintsch, W. (1998). Comprehension. A paradigm for cognition. Cambridge, England: Cambridge University Press.

Kirby, J. R. \& Savage, R. S. (2008). Can the simple view deal with the complexities of reading? Literacy, 42, 75-82. doi: 10.1111/j.1741-4369.2008.00487.x

Oliveira, K. L., Boruchovitch, E. \& Santos, A. A. A. (2007). A técnica de Cloze na avaliação da compreensão em leitura. In A. A. A. Santos, E. Burochovitch \& K. L. Oliveira (Orgs.), Cloze: um instrumento de diagnóstico e intervenção ( $\mathrm{pp}$. 47- 78). São Paulo: Casa do Psicólogo.

Perfetti, C. A. (1985). Reading ability. New York: Oxford University Press

Savage, R. (2006). Reading comprehension is not always the product of nonsense word decoding and linguistic comprehension: Evidence from teenagers who are extremely poor readers. Scientific Studies of Reading, 10, 143-164.

Seymour, P. H. K., Aro, M., \& Erskine, J. M. (2003). Foundation literacy acquisition in European orthographies. British Journal of Psychology, 94, 143-174.

Solé, I. (1992). Estratégias de leitura. São Paulo: Artmed.

Stein, L. M. (1994). TDE - Teste de desempenho escolar: Manual para aplicação e interpretação. Itatiba: Casa do Psicólogo.

\section{Endereço para correspondência \\ Marcia Oliveira}

Universidade Salgado de Oliveira - UNIVERSO

Rua Marecha Deodoro, 217, Centro, CEP 24.030-060, Niterói - RJ, Brasil

Endereço eletrônico: marciaosilva@gmail.com

Márcia Maria Peruzzi Elia da Mota

Universidade Salgado de Oliveira - UNIVERSO

Rua Marecha Deodoro, 217, Centro, CEP 24.030-060, Niterói - RJ, Brasil

Endereço eletrônico: mmotapsi@gmail.com

Recebido em: 13/11/2015

Reformulado em: 28/04/2016

Aceito em: $26 / 10 / 2016$ 


\section{Notas}

* Pós-doutoranda pelo programa de Psicologia da Universidade Salgado de Oliveira. Doutora em Psicologia (UNIVERSO, 2016), Docente na Universidade Salgado de Oliveira, professora na rede pública de ensino no Rio de Janeiro, graduada em Ciências biológicas.

** Doutora em Psicologia na Universidade de OXFORD. Docente do Programa de Pós-graduação da Universidade Salgado de Oliveira e da Universidade do Estado do Rio de Janeiro, Diretora do Instituto de Psicologia da Universidade do Estado do Rio de Janeiro. Atua na área de psicologia com ênfase em processos perceptuais e cognitivos; e desenvolvimento humano. 